Louisiana State University

LSU Digital Commons

$2-11-2013$

\title{
Enhancing entangled-state phase estimation by combining classical and quantum protocols
}

\author{
Heedeuk Shin \\ Sandia National Laboratories, New Mexico \\ Omar S. Magaña-Loaiza \\ University of Rochester Institute of Optics \\ Mehul Malik \\ University of Rochester Institute of Optics \\ Malcolm N. O'Sullivan \\ University of Rochester Institute of Optics \\ Robert W. Boyd \\ University of Rochester Institute of Optics
}

Follow this and additional works at: https://digitalcommons.Isu.edu/physics_astronomy_pubs

\section{Recommended Citation}

Shin, H., Magaña-Loaiza, O., Malik, M., O'Sullivan, M., \& Boyd, R. (2013). Enhancing entangled-state phase estimation by combining classical and quantum protocols. Optics Express, 21 (3), 2816-2822.

https://doi.org/10.1364/OE.21.002816

This Article is brought to you for free and open access by the Department of Physics \& Astronomy at LSU Digital Commons. It has been accepted for inclusion in Faculty Publications by an authorized administrator of LSU Digital Commons. For more information, please contact ir@lsu.edu. 


\title{
Enhancing entangled-state phase estimation by combining classical and quantum protocols
}

\author{
Heedeuk Shin, ${ }^{1, *}$ Omar S. Magaña-Loaiza, ${ }^{2}$ Mehul Malik, ${ }^{2}$ Malcolm \\ N. O'Sullivan, ${ }^{2}$ and Robert W. Boyd ${ }^{2,3}$ \\ ${ }^{1}$ Sandia National Laboratories, Albuquerque, New Mexico 87123 USA \\ ${ }^{2}$ The Institute of Optics, University of Rochester, Rochester, New York 14627 USA \\ ${ }^{3}$ Department of Physics, University of Ottawa, Ottawa, ON K1N 6N5 Canada \\ *heedeuk.shin@gmail.com
}

\begin{abstract}
Here we describe a laboratory procedure by which we have increased the resolution of a measurement of the position of an optical component by a factor of 16 . The factor of 16 arises from a four-fold quantum enhancement through the use of an $N=4 N 00 N$ state and a four-fold classical enhancement from a quadruple pass through a prism pair. The possibility of achieving supersensitivity using this method is discussed.
\end{abstract}

\section{(C) 2013 Optical Society of America}

OCIS codes: (120.5050) Phase measurement; (120.3180) Interferometry; (270.5585) Quantum information and processing; (120.3940) Metrology.

\section{References and links}

1. V. Giovannetti, S. Lloyd, and L. Maccone, "Advances in quantum metrology," Nat. Photonics 5(4), 222-229 (2011).

2. A. N. Boto, P. Kok, D. S. Abrams, S. L. Braunstein, C. P. Williams, and J. P. Dowling, "Quantum interferometric optical lithography: exploiting entanglement to beat the diffraction limit," Phys. Rev. Lett. 85(13), 2733-2736 (2000).

3. M. W. Mitchell, J. S. Lundeen, and A. M. Steinberg, "Super-resolving phase measurements with a multiphoton entangled state," Nature 429(6988), 161-164 (2004).

4. P. Walther, J. Pan, M. Aspelmeyer, R. Ursin, S. Gasparoni, and A. Zeilinger, "de Broglie wavelength of a nonlocal four-photon state," Nature 429(6988), 158-161 (2004).

5. T. Nagata, R. Okamoto, J. L. O'Brien, K. Sasaki, and S. Takeuchi, "Beating the standard quantum limit with four-entangled photons," Science 316(5825), 726-729 (2007).

6. D. V. Korobkin and E. Yablonovitch, "Two-fold spatial resolution enhancement by two-photon exposure of photographic film," Opt. Eng. 41, 1729-1732 (2002).

7. N. Thomas-Peter, B. Smith, A. Datta, L. Zhang, U. Dorner, and I. Walmsley, "Real-world quantum sensors: evaluating resources for precision measurement," Phys. Rev. Lett. 107(11), 0113603 (2011).

8. G. M. Gehring, H. Shin, R. Boyd, C. M. Kim, and B. S. Ham, "Tunable optical time delay of quantum signals using a prism pair," Opt. Express 18(18), 19156-19162 (2010).

9. B. C. Barish and R. Weiss, "LIGO and the detection of gravitational waves," Phys. Today 52(10), 44-50 (1999).

10. F. Zernike, "Phase contrast, a new method for the microscopic observation of transparent objects," Physica 9(7), 686-698 (1942).

11. F. Marquardt and S. Girvin, "Optomechanics," Physics 2, 40 (2009).

12. B. L. Higgins, D. W. Berry, S. D. Bartlett, H. M. Wiseman, and G. J. Pryde, "Entanglement-free Heisenberglimited phase estimation," Nature 450(7168), 393-396 (2007).

13. S. Braunstein and C. Caves, "Statistical distance and the geometry of quantum states," Phys. Rev. Lett. 72(22), 3439-3443 (1994).

14. R. A. Fisher, "Theory of statistical estimation," Mathematical Proceedings of the Cambridge Philosophical Society 22(05), 700-725 (1925). 
15. K. J. Resch, K. L. Pregnell, R. Prevedel, A. Gilchrist, G. J. Pryde, J. L. O’Brien, and A. G. White, “Time-reversal and super-resolving phase measurements," Phys. Rev. Lett. 98(22), 0223601 (2007).

16. R. Campos, B. Saleh, and M. Teich, "Quantum-mechanical lossless beam splitter: SU(2) symmetry and photon statistics,” Phys. Rev. A 40(3), 1371-1384 (1989).

17. H. Shin, K. W. C. Chan, H. J. Chang, and R. W. Boyd, "Quantum spatial superresolution by optical centroid measurements," Phys. Rev. Lett. 107, 083603 (2011).

18. O. Steuernagel, "de Broglie wavelength reduction for a multiphoton wave packet," Phys. Rev. A 65, 033820 (2002).

19. J. U. White, "Long optical paths of large aperture," J. Opt. Soc. Am. 32(5), 285-288 (1942).

\section{Introduction}

The ability to accurately measure small variation of physical parameters with limited amount of resource has been of fundamental importance in classical interferometry and metrology. Unfortunately, quantum mechanics and the nature of classical light impose a well defined limit on the precision that can be achieved in such a measurement. This limit is known as the standard quantum limit (SQL). In the context of precision measurements of phase, this is represented by the phase uncertainty $\Delta \phi_{S Q L}=1 / \sqrt{n}$, where $n$ is the the mean number of photons involved in performing the measurement. It is possible to beat this classical limit by using nonclassical light, leading to a phenomenon known as supersensitivity [1]. However, the precision in the measurement of phase with nonclassical light is also fundamentally limited. This limit, commonly referred to as the Heisenberg limit, is given by $\Delta \phi_{H L}=1 / n$.

A wide variety of experiments have been proposed with the aim to overcome the SQL. Many of these experiments are based on the generation and detection of $N 00 N$ states of light [2-5]. In spite of the their vulnerability to loss and difficult methods of generation and detection, $N 00 \mathrm{~N}$ states have became a key resource for quantum interferometric lithography, quantum imaging and quantum metrology. Initially it was thought that the "superresolved" nature of interference patterns obtained beyond the Rayleigh diffraction limit was due to the quantum nature of $N 00 \mathrm{~N}$ states. However, Korobkin et. al. soon demonstrated that superresolution can be achieved with classical light [6]. On the other hand, phase supersensitivity is only exhibited in the case of quantum interference.

Recently, Thomas-Peter et. al. showed that the phase measurement sensitivity in $N 00 N$ state interference is strongly dependent on experimental imperfection [7]. The delicate nature of $N 00 N$ states and real-world detector efficiencies makes the majority of the $N$-photon detection events lost. Further, non-unit fringe visibility decreases the measurement accuracy, and the visibility can be used to identify whether a superresolved interference pattern exhibits phase supersensitivity. In practice, this imperfection of system establishes experimental boundaries which make it essentially difficult to beat the SQL with $N 00 N$ states.

In this paper, we show that the sensitivity of entangled-state phase estimation can be enhanced more by combining quantum and classical protocols. We demonstrate a novel experimental technique that allows us to increase the resolution of a phase measurement by a factor of 16. In order to achieve this, we combine a quantum interferometric method with multiple passes through a prism pair [8]. The quantum method gives us a four-fold enhancement of resolution through the use of an $N=4 N 00 N$ state. The $N O O N$ state undergoes four passes through a prism pair, which gives us an additional four-fold enhancement of resolution. In addition, the experimental data analysis shows that our phase measurement accuracy is also increased. The precision of our phase measurement strongly depends on the system loss, such as the absorption and reflection of optics as well as imperfect detection efficiency. We discuss the phase measurement precision achieved with our method and compare it with that obtained without. This method could find applications in phase variation measurements for the detection of gravitational waves [1,9], in bio-imaging [10], and in nano-scale optomechanics [11].

\#180368 - \$15.00 USD

(C) 2013 OSA
Received 21 Nov 2012; revised 9 Jan 2013; accepted 9 Jan 2013; published 29 Jan 2013 11 February 2013 / Vol. 21, No. 3 / OPTICS EXPRESS 2817 


\section{Theory}

We first describe the resolution enhancement that is achieved in a Mach-Zehnder interferometer through the use of $N 00 N$ states. Assume a variable phase retarder is located in mode $A$ of the interferometer. Each photon existing in this mode will experience a phase shift $\phi$. As the phase of the retarder is varied, a classical interference pattern is obtained in the output ports of the interferometer. The intensity of this pattern is modulated by one period for a phase shift of $2 \pi$, and is described by the sinusoidal function $\cos ^{2}(\phi / 2)$.

When the $N 00 N$ state $\left(|N\rangle_{A}|0\rangle_{B}+|0\rangle_{A}|N\rangle_{B}\right)$ is input into the interferometer, the net phase acquired by the $N$ photons results in the output state $\left(e^{-i N \phi}|N\rangle_{A}|0\rangle_{B}+|0\rangle_{A}|N\rangle_{B}\right) . N$-photon detection at the output of the interferometer gives an interference pattern described by the sinusoidal function $\cos ^{2}(N \phi / 2)$ [2]. It is clear that the resolution of the resulting interference fringes is increased by a factor of $N$. This resolution enhancement can be mimicked by using classical protocol of multiple reflections. If we introduce multiple passes in the arm of the interferometer containing the variable phase retarder, a further enhancement of the resolution can be achieved [12]. When the photons pass $M$ times through the same variable phase retarder, they will acquire a net phase of $M \phi$. Furthermore, these quantum and classical protocols can be combined. If a $N 00 N$ state is input into such an interferometer, the output state will have the form $\left(e^{-i N M \phi}|N\rangle_{A}|0\rangle_{B}+|0\rangle_{A}|N\rangle_{B}\right)$, and the interference pattern will be described by the function $\cos ^{2}(N M \phi / 2)$ showing that the resolution is increased by a factor of $N M$, compared with that of single-pass classical-light interference.

In quantum mechanics, a variable phase retarder can be described by using the unitary operator $\mathrm{U}(\phi)=\exp (-i \phi H)$, where $H$ represents the phase transformation operator $H=\hat{a}^{\dagger} \hat{a}$ [13]. Here, $\hat{a}$ and $\hat{a}^{\dagger}$ represent annihilation and creation operators respectively. The output state is then related to the input state by the equation $\Psi(\phi)=\mathrm{U}(\phi) \Psi(0)$. In quantum estimation theory, the phase uncertainty $\Delta \phi$ is related to the quantum Fisher information [14] $F_{Q}(\phi)$ as $\Delta \phi \geq 1 / \sqrt{q F_{Q}(\phi)}$, where $q$ is the number of repeated measurements [13].

The quantum fisher information for a coherent state in an interferometer is related to the amplitude of the state as $F_{Q}(\phi)=\left|\alpha_{0}\right|^{2}$. This is equivalent to the average number of photons, $\bar{n}$. The uncertainty in phase for such a system is then given by the relation $\Delta \phi \geq 1 / \sqrt{q \bar{n}}$. For a coherent state in an interferometer with $M$ multiple passes in one arm, the quantum fisher information is increased to $F_{Q}(\phi)=M^{2} \bar{n}$, resulting in a lower bound for the uncertainty in phase $\Delta \phi \geq 1 /(M \sqrt{q \bar{n}})$. This enhancement in sensitivity can be applied to a $N 00 N$ state interferometer by adding $M$ multiple passes in an interferometer arm, further lowering the phase uncertainty bound. The quantum fisher information for such a system is given by $F_{Q}(\phi)=(N M)^{2}$. This results in an even lower bound for the uncertainty in phase given by $\Delta \phi_{(N, M)} \geq 1 /(N M \sqrt{q})$. Thus, we see that adding $M$ multiple passes in a $N 00 N$ state interferometer enhances the resolution and phase sensitivity of the system by a factor of $M$.

In reality, experimental imperfections such as system loss and non-unit detector efficiency have a strong effect on the phase sensitivity. Further, the non-unit visibility will linearly reduce the accuracy of the phase measurement $[5,15]$. In addition, one must take into account the intrinsic $N 00 N$ state generation efficiency, which is the ratio of the number of photons generated in a particular $N 00 N$ state to the $N$-photon interference [5]. For $N=2 N 00 N$ state, this efficiency is equal to one. However, for $N>2 N 00 N$ states, the generation efficiency is reduced to lower than one $[5,16]$. We elaborate on the generation efficiency of our $N=4 N 00 N$ state in the next section. Taking these factors into account, our uncertainty in phase is now defined as

$$
\Delta \phi_{(N, M)} \geq 1 /\left(N M V \sqrt{\eta_{g} \eta_{\text {net }} q}\right)
$$

where $V$ is the visibility of the interference pattern and $\eta_{g}$ is the intrinsic $N 00 N$ state generation efficiency. $\eta_{\text {net }}$ is the net system efficiency and is given by $\eta_{\text {net }}=\left(\eta_{s y s} \eta_{p p}^{M} \eta_{\text {det }}\right)^{N}$. Here, $\eta_{\text {sys }}$ is 

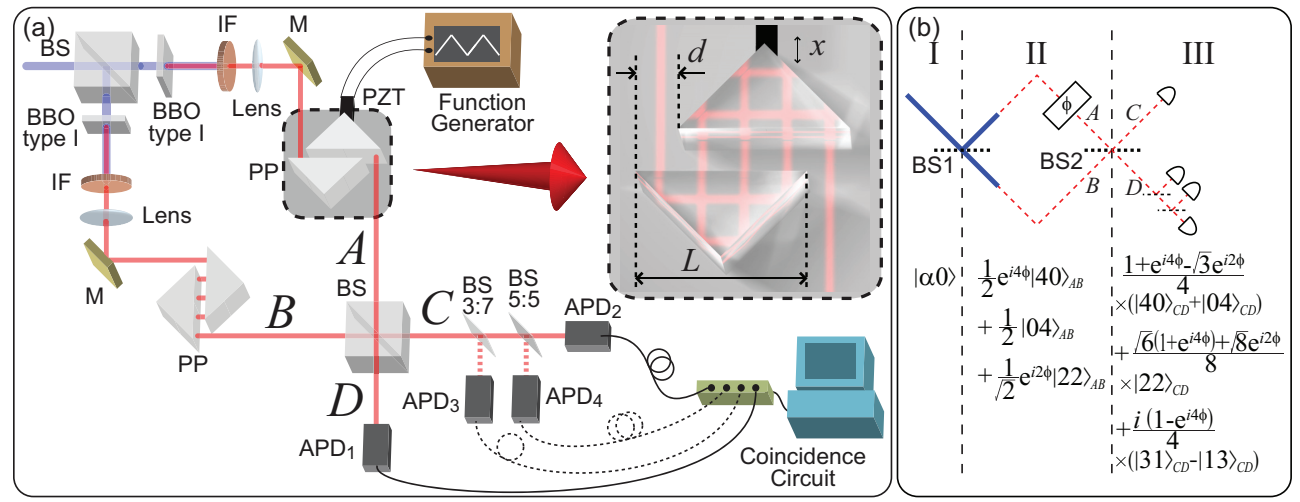

Fig. 1. (a) Schematic of the experimental setup. BS: non-polarizing beam splitter, IF: interference filter, PZT: Piezoelectric actuator, PP: prism pair, and APD: detector. Inset: The detailed prism pair alignment and the beam path for $\mathrm{M}=2$ through the prism pair. $\mathrm{d}$ : the lateral displacement of two prisms, $\mathrm{x}$ : the longitudinal movement of a prism, and L: the hypotenuse length. (b) Simplified schematic of the setup, and the four-photon states at each stage I, II, and III. $\phi$ is the phase difference between two paths, $A$ and $B$.

the optical transmittance of the interferometer, $\eta_{p p}$ is the single-pass transmittance of the prism pair, and $\eta_{\text {det }}$ is the detector efficiency.

\section{Experimental setup}

A proof-of-principle experiment was performed by using a Mach-Zehnder interferometer as shown in Fig. 1(a). Second harmonic Femto-second pump pulses at $400 \mathrm{~nm}$ were split into two paths $A$ and $B$ at a beam splitter (BS) and each beam was incident on a $\beta$-Barium Borate (BBO) crystal. Energy-time entangled photon pairs were randomly generated in each BBO crystal by the process of spontaneous parametric down conversion (SPDC) under type-I collinear phase matching conditions [17]. The pump power in both paths was adjusted to generate probabilistically equal numbers of photon pairs. The input state at stage I of Fig. 1(b) is a coherent pump-photon state, and the generated two-and four-photon number states in paths $A$ and $B$ after the interference filters (IF) are probabilistically given by

$$
\begin{aligned}
& \left|\Psi_{2}\right\rangle=\frac{1}{\sqrt{2}}\left(e^{i 2 \phi}|20\rangle_{A B}+|02\rangle_{A B}\right) \\
& \left|\Psi_{4}\right\rangle=\frac{1}{2}\left(e^{i 4 \phi}|40\rangle_{A B}+|04\rangle_{A B}\right)+\frac{1}{\sqrt{2}} e^{i 2 \phi}|22\rangle_{A B},
\end{aligned}
$$

where $\phi$ is the phase difference between two paths, $A$ and $B$. Note that the two-photon $N 00 N$ state has unit intrinsic efficiency, but that of the four-photon $N 00 \mathrm{~N}$ state is $1 / 4$ due to the unwanted $|22\rangle_{A B}$ term in equation 3. The four-photon state at stage III in Fig. 1(b) has $|13\rangle_{C D}$ state with the amplitude of $i\left(1-e^{i 4 \phi}\right) / 4$ indicating that quantum interference cancels the contribution from $|22\rangle_{A B}$ state to $|13\rangle_{C D}$ state via post-selection $[5,18]$. The beam splitters BS3:7 and BS5:5 and detectors APD3 and APD4 are additionally used for measuring post-selected four-photon events. The intrinsic efficiency of the four-photon $N 00 \mathrm{~N}$ state in this paper can be enhanced by a factor of 1.5 by using the four-photon creation method described in Ref. [5]. A lens is located in each path to increase the collection efficiency of the photons. A prism pair (PP) with a piezo actuator (PZT) on one prism is located in path $A$. Another prism pair is placed in path $B$ for the purpose of path-length matching. A triangular waveform voltage is applied to 
the actuator with a repetition rate of $0.004 \mathrm{~Hz}$. The prism on the piezo actuator moves by 400 $\mathrm{nm}$ for a voltage change of $4.8 \mathrm{~V}$. Both beams are combined at the second beam splitter (BS), and the output from the beam splitter is measured by single-photon counting detectors (APDs) with a coincidence circuit. The coincidence circuit has a time windows of $7 \mathrm{~ns}$ for two-photon coincidences and $5.4 \mathrm{~ns}$ for four-photon coincidences. The integration times used for detecting single-, two- and four-photon coincidence counts were $0.2,0.2$, and 100 seconds, respectively.

The beam path inside the prism pair for $M=2$ passes is shown in the inset of Fig. 1. Photons entering through the hypotenuse of a right angle prism will undergo multiple reflections and exit the prism also through the hypotenuse in a direction parallel to the input path [8]. The number of reflections $M$ through the prism pair is related to the hypotenuse length $L$ and the displacement parameter $d$ according to $M=\operatorname{round}(L / 2 d)$. The prism pair has the practical advantage of relatively simple alignment when changing the number of passes $M$ through the two prisms, as compared to two parallel/concave mirrors $[12,19]$.

\section{Experimental results}

Interference patterns obtained with our interferometer are shown in Fig. 2. Single-photon counts are plotted as a function of piezo voltage in Fig. 2(a). These show the interference pattern obtained by sending a strongly attenuated coherent state at $800 \mathrm{~nm}$ through the interferometer in Fig. 1 without multiple passes through the prism pairs $(M=1)$. Figures 2(b)-2(c) show measured two-photon coincidence count rates as a function of piezo voltage. These exhibit interference patterns obtained with an $N=2 N 00 N$ state for different numbers of multiple passes $M=2$ and 4 through the prism pairs. Figure 2(d) shows the measured four-photon interference pattern for $M=4$ multiple passes through the prism pair, and Fig. 2(e) is a zoomedin plot of Fig. 2(d). The resolution enhancement is apparent in Figs. 2(a)-2(d) and is clearly seen to be proportional to the product of the entangled photon number $N$ and the number of multiple passes $M$ through the prism pairs. Specifically, the four-photon interference pattern with a quadruple pass through the prism pair (Fig. 2(d)) exhibits a resolution enhancement by a factor of 16 compared with that of the single-photon interference pattern with single pass $(\bar{n}$ $=M=1)$. These superresolution results clearly show that the resolution of interference patterns can be enhanced by combining $N 00 N$ state interferometry with a simple double prism setup.

Next, we analyze our data to determine the accuracy in phase measurement. A sinusoidal fit made to the data in Figs. 2(a)-2(d) shows visibilities $V$ of $85 \%, 70 \%, 65 \%$, and 40\%, respectively. Taking in account the efficiency of our single photon detectors $\left(\eta_{\text {det }}=0.62\right)$ and the ratio between measured single and two-fold coincidence counts (6.3\%), the net detection efficiency of our system $\eta_{\text {net }}$ is estimated to be 0.25 for single photon detection assuming identical losses in both pathes. The transmission loss due to multiple passes through the prism pair is negligible due to the use of anti-reflection coatings on the prisms used. The minimum phase uncertainty (in radians) for a coherent state with no multiple pass through the prism pairs is then calculated to be $\Delta \phi=1 /\left(V \sqrt{\eta_{\text {net }} \bar{n} q}\right)=1 /(0.85 \cdot \sqrt{0.25 \cdot 1})=2.35$. In contrast, the minimum phase uncertainty for an $N=2 N 00 N$ state with $M$ passes through the prism pairs is given by Eq. (2) as $\Delta \phi_{(N, M)}=1 /\left(N M V \sqrt{\eta_{g} \eta_{\text {net }} q}\right)$. Here, $\eta_{\text {net }}=(0.25)^{2}=0.0625$, as we are measuring two-photon events. The minimum phase uncertainty in Figs. 2(b)-2(c) is then calculated to be $\Delta \phi_{(2,2)}=1.4$ and $\Delta \phi_{(2,4)}=0.8$.

For the $N=4 N 00 N$ state, note that additional beam splitters BS3:7 \& BS5:5 and detectors APD3 \& APD4 are added in front of APD2 reducing the system efficiency of APD2, APD3, and APD4 by $1 / 3$ when compared to that of APD1. Then the net system efficiency is very low $\left(\eta_{\text {net }}=0.25^{4} / 3^{3}=0.000145\right)$. This low net system efficiency as well as low visibility and non-unit intrinsic efficiency for the four-photon interference has a strong effect on the minimum phase uncertainty for the case shown in Fig. 2(d), which is calculated to be $\Delta \phi_{(4,4)}=26.0$.

$\# 180368$ - \$15.00 USD

(C) 2013 OSA
Received 21 Nov 2012; revised 9 Jan 2013; accepted 9 Jan 2013; published 29 Jan 2013 11 February 2013 / Vol. 21, No. 3 / OPTICS EXPRESS 2820 


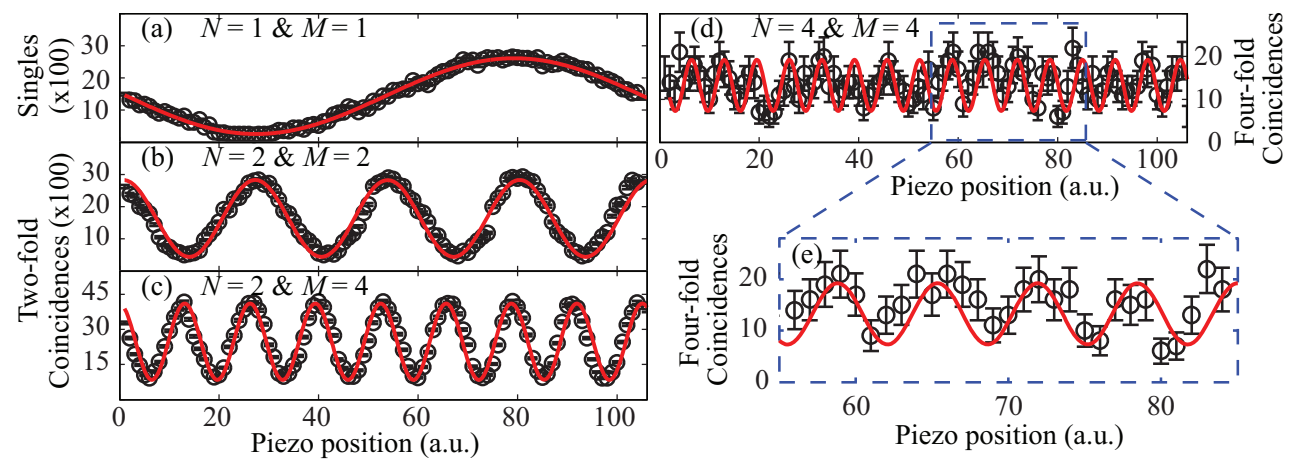

Fig. 2. Experimental data showing interference patterns obtained as a function of piezo voltage. (a) Single-photon count rates for a strongly attenuated coherent state at $800 \mathrm{~nm}$ without any passes through the prism pair $(M=1)$. Two-photon count rates for the $N=2$ $N 00 N$ state with (b) $M=2$ and (c) $M=4$ multiple passes through the prism pair. (d) Fourphoton count rates for the $N=4 N 00 N$ state for $M=4$ multiple passes through the prism pair, and (e) the zoomed-in plot of the dashed box in (d). The interference patterns in (a)-(d) were performed under identical experimental conditions. The solid curves are theoretical fits to the data. Error bars show the standard deviation of counts $( \pm \sqrt{\text { counts }})$.

From these results, it is clear that we are able to enhance the phase sensitivity of our interferometer by introducing multiple passes through a prism pair. Unfortunately, the non-unit detector $\eta_{\text {det }}$ and system $\eta_{s y s}$ efficiencies significantly lower the accuracy in phase measurement as we go to a larger number of multiple-photon events $N$. The use of multiple passes in the interferometer arms can be used to ameliorate the effect of low detection efficiency on the phase sensitivity. This is seen clearly as we increase the number of multiple reflections in the $N=2 N 00 N$ state interferometer from 2 to 4 . The minimum phase sensitivity is lowered from 1.4 to 0.8 in this case. In principle, we could apply a similar tactic to the $N=4 N 00 N$ state interferometer to achieve high phase sensitivity in addition to superresolution.

\section{Conclusions}

We have performed an experiment showing an enhancement in resolution and phase sensitivity of a quantum interferometric method with the simple addition of a prism pair. We achieve an interference pattern that displays a resolution enhancement by a factor of 16 when compared to a traditional Mach-Zehnder interferometer. The factor of 16 comes from a quantum enhancement of 4 through the use of an $N=4 N 00 N$ state and a classical enhancement of 4 from a quadruple pass through a prism pair. Further, we are able to show that the addition of multiple passes in the arms of the interferometer leads to a more accurate phase measurement than without. However, we do not achieve phase supersensitivity due to the fact that the sensitivity in phase is strongly dependent on our net system efficiency [7]. This is severely limited by imperfect detectors, system transmission, and $N 00 \mathrm{~N}$ state generation efficiency. In order to achieve phase supersensitivity, we would need to obtain unit fringe visibility and increase our net system efficiency to 0.707 for an $N=2 N 00 N$ state. To achieve this criterion with an $N=4$ $N 00 N$ state by our current experimental apparatus, we would need unit fringe visibility and a unit net system efficiency. We are working towards improving our interference visibility and net system efficiency for future experiments.

(C) 2013 OSA
Received 21 Nov 2012; revised 9 Jan 2013; accepted 9 Jan 2013; published 29 Jan 2013

11 February 2013 / Vol. 21, No. 3 / OPTICS EXPRESS 2821 


\section{Acknowledgments}

Sandia Laboratory is operated by Sandia Co., a Lockheed Martin Company, for the U.S. Department of Energy's NNSA under Contract No. DE-AC04-94AL85000. This work was supported by the DARPA InPho program. OSML acknowledges support from the CONACyT of Mexico.

\#180368 - \$15.00 USD

(C) 2013 OSA 